\title{
EDITORIAL
}

\section{El ejercicio clínico en endocrinología: Retos futuros en el sistema de salud para Colombia}

$\mathrm{F}$ ernando Anzures, autor mexicano de varios escritos y líder de una gran empresa de consultoría en $\mathrm{Ma}$ nagement, definió los cinco factores que mueven los hilos de la influencia positiva en cualquier sistema social: autoridad de los que la lideran, consistencia en las acciones de todos, consenso en las decisiones, reciprocidad en el actuar y empatía en las relaciones.

Desafortunadamente, en la actualidad, ninguno de esos factores se aplica en el sector salud en Colombia; podríamos decir que el sentimiento es de desconfianza entre los diferentes agentes del sistema: los prestadores desconfían de las EPS; los médicos desconfían del sistema; la mayoría desconfía del Estado y en medio de todos se encuentra el paciente, centro vital de nuestro quehacer y cuyo único objetivo (el del paciente y por extensión el de su familia) es que su dolor y sufrimiento se conjuguen de una manera rápida, efectiva y humana.

Equivocada y apresuradamente creemos que para el sistema de salud no hay salida; muchas veces juzgamos la realidad con los anhelos y añoranzas de un mejor pasado y se nos olvida que debemos construir el futuro de una labor bendita, imprescindible, trascendente y de alto impacto social como lo es la Medicina, y lo peor es que a veces no conocemos bien el teatro y el contexto en el que se desenvuelve nuestro actuar.

En este orden de ideas, el presente escrito trata de dar los elementos principales de una realidad que ya estamos viviendo: un sistema de salud que, a pesar de sus imperfecciones, con desaciertos en su operación, a veces alejado de la realidad y con un énfasis más financiero y económico, y que en ocasiones olvida el dolor del ser humano, es el que nos rige; que en su esencia es bien calificado por propios y ajenos; pero que en su día a día pasa de ser desafiante a ser un fiasco. Vamos a recorrer con el mayor respeto el camino de los cambios que se vienen a futuro y a describir una realidad, dando el marco de desenvolvimiento para que la labor médica, en una rama trascendente como lo es la Endocrinología, sea más efectiva y llegue positivamente al foco de actuación: el paciente.

\section{¿Qué es lo nuevo del sistema?}

Son tres las normas protagonistas, principales y esenciales en el ejercicio médico: la Ley Estatutaria de la Salud, el Modelo Integral de Atención en salud y la Resolución del proceso de financiación de las actividades no cubiertas por el Plan de beneficios.

1. La Ley Estatutaria en Salud define el derecho fundamental a la salud de los colombianos, esto es que no se puede negar a ningún colombiano el acceso a la salud a través de un servicio oportuno, eficaz y de calidad; la ley es de obligatorio cumplimiento por parte del Estado. Busca garantizar la atención integral a través de la promoción, prevención, diagnóstico, tratamiento, recuperación, rehabilitación y paliación de una enfermedad. El derecho a la salud de los colombianos se complementa con un deber de autocuidado, lo que implica que el primer factor de verdadera solidaridad de los pacientes con la sostenibilidad del sistema se sustenta en tener hábitos saludables de vida y seguir todas las indicaciones sugeridas por los servicios de salud.

Otros dos puntos fundamentales que se desarrollan en el marco de la Ley Estatutaria son, en primera instancia, la autonomía que tendrán los profesionales de la salud en su práctica rutinaria y en sus decisiones al momento de tratar a un paciente. Sin embargo, la autonomía como derecho se equilibra con un factor: el 
deber de autocontrolarse y buscar la mayor eficiencia en la práctica profesional; la Ley también indica que los profesionales de la salud pueden ser sancionados en "caso de constreñimiento, soborno o cualquier abuso en su ejercicio profesional, que atente contra la salud del paciente".

El segundo punto que se debe resaltar es la eliminación de las actividades, procedimientos, medicamentos e insumos del Plan Obligatorio de Salud: "La Ley Estatutaria de Salud acaba con la lista de servicios de salud que estaban en el POS y a los que anteriormente los pacientes podían acceder. Con la Ley 1751 de 2015 los pacientes pueden acceder a todos los servicios necesarios para su recuperación, con excepción de tratamientos que tengan una finalidad cosmética, que no cuenten con evidencia científica sobre su efectividad, eficiencia y seguridad clínica; que estén en fase de experimentación y que se tengan que prestar en el exterior".

Igualmente la Ley garantiza unas condiciones laborales justas y dignas para los médicos, como estabilidad y facilidad para incrementar sus conocimientos.

En este orden de ideas, los especialistas y subespecialistas clínicos deben identificar su papel y oferta de servicios en el marco de la integralidad. Procesos como la prevención de la enfermedad, promoción de estilos sanos de vida y paliación del sufrimiento, pueden ser alternativas importantes para ofrecer servicios que, en efecto, resuelvan necesidades globales de pacientes y familias. Estos servicios antaño eran reservados a organizaciones y profesionales de la atención básica que normalmente no se articulaban a los servicios de especialidad.

Además, los servicios de endocrinología deben iniciar la medición sistemática de resultados en salud. Indicadores clínicos, paraclínicos, sociales, de calidad de vida, de percepción y algunos de relacionamiento, deben ser de rutinaria evaluación. Normalmente se deben utilizar mediciones cuantitativas; sin embargo, las evaluaciones cualitativas son alternativas que a veces resultan apartadas, más por desconocimiento que por convicción.

Finalmente, los profesionales de la salud deben fortalecer los procesos de comunicación y orientación de los pacientes. La correcta interacción, además de buscar el autocuidado, es un verdadero valor agregado y diferencial positivo para los servicios; deben ofertarse formas de orientación más allá de los rutinarios escritos proforma o de los videos institucionales.

2. Política Integral de Atención en Salud. Su objetivo está dirigido hacia la "generación de las mejores condiciones de salud en la población, mediante la regulación de la intervención de los integrantes sectoriales e intersectoriales responsables de garantizar la atención de la promoción, prevención, diagnóstico, tratamiento, rehabilitación y paliación en condiciones de accesibilidad, aceptabilidad, oportunidad, continuidad, integralidad y capacidad de resolución".

Para esto se definen diez componentes del Modelo Integral de Atención en Salud- MIAS-, que incluyen las acciones que se deben implementar, entre las que se destacan la caracterización de la población, entendido esto como la necesidad de organizar las poblaciones en grupos de riesgo; definir las Rutas Integrales de Atención en Salud, que determinan los momentos, actividades y procesos que generen un cuidado integral del individuo en cada entorno y que incluyen las intervenciones para la prevención, diagnóstico, tratamiento, rehabilitación de la enfermedad y la implementación de la Gestión Integral del Riesgo en Salud -GIRS- que comprende la coordinación, monitoreo y ajuste de todas las acciones intencionales y planificadas dentro de cada grupo de riesgo. Las intervenciones están dirigidas a anticiparse a la enfermedad.

Dentro de los componentes, toma vital importancia la definición de las Redes Integrales de Prestadores de Servicios de Salud. Una Red Integral de Prestación de Servicios (RED) se define como el conjunto articulado de prestadores de servicios de salud u organizaciones funcionales de servicios de salud, públicos y privados, ubicados en un ámbito territorial definido de acuerdo con las condiciones de operación del MIAS, con una organización funcional que comprende un componente primario y un componente complementario. El componente primario son los servicios que eran denominados de baja complejidad y el componente complementario es el equivalente a la alta complejidad, en donde se encuentra el ejercicio de la Endocrinología.

Es importante que los endocrinólogos logren su posición en la oferta coherente de servicios de los diferentes agentes de salud como son las IPS y las EPS. La ubicación puede determinarse en acciones de prevención, 
tratamiento y rehabilitación. La participación en la implementación de las rutas integrales de atención son de suma relevancia.

La Asociación de Endocrinología debería proponer y proyectar el mejoramiento de las condiciones laborales, fortalecer las competencias y desarrollar proyectos de investigación.

3. Implementación del aplicativo WEB -MIPRES-. Es la reglamentación del procedimiento de acceso, reporte de prescripción, garantía del suministro, verificación, control, pago y análisis de la información de servicios y tecnologías en salud no cubiertas por el Plan de Beneficios en Salud con cargo a la UPC. La norma fue promulgada en abril del año anterior y fundamenta sus lineamientos en la Ley Estatutaria y su supuesto de que "El sistema de salud debe procurar por la mejor utilización social y económica de los recursos, servicios y tecnologías disponibles para garantizar el derecho a la salud de toda la población”.

En esencia, la Resolución reglamenta un procedimiento. Caracteriza la manera como se prescriben, registran, dispensan, facturan y cobran los medicamentos, procedimientos, insumos, productos nutricionales y elementos complementarios que buscan resolver una necesidad de salud de los pacientes. Brinda los tiempos, roles y responsabilidades de los diferentes agentes del sistema de salud en el proceso.

Su ámbito de aplicación es esencialmente para el régimen contributivo pero por voluntad de departamentos y distritos se podría aplicar para el régimen subsidiado. Está vigente y media un aplicativo WEB para el diligenciamiento de la solicitud, lo que implica un mínimo de condiciones tecnológicas en las IPS y en las EPS; elimina de tajo el CTC, controvertido y a veces odioso; supone la autorregulación y el autocontrol y requiere algunas condiciones que permitan que los médicos y otros profesionales de la salud puedan convertirse en prescriptores "autorizados".

El médico -para el caso el endocrinólogo- debe asegurarse que está inscrito en el RETHUS, sistema de registro del talento humano en salud; la verificación es sencilla y se hace en línea. El profesional no inscrito debe hacerlo a través de la instancia autorizada normativamente y las sociedades científicas deben asegurar la inscripción como especialista (normalmente la inscripción se hace como médico general). Paralelamente, los médicos deben registrarse como personas naturales, para que sean potencialmente prescriptores, proceso muy sencillo y que arroja un usuario y una clave única e intransferible -que llega al correo registrado-y que es la que se usa para prescripción en cualquier lugar de trabajo del profesional.

Finalmente, cada IPS debe registrar los prescriptores (previa inscripción como persona jurídica) y las EPS deben registrar su red de prestación que vinculará a IPS (a través de sus médicos) y consultorios como potenciales formuladores.

El aplicativo WEB (que seguramente va a tener dificultades en los primeros meses de su implementación) lleva la información de la prescripción a la EPS de manera automática para que lo prescrito llegue en efecto al paciente.

El principal reto para los médicos es la autorregulación: al paciente se le debe dar lo que sea necesario, SOLO cuando sea necesario. Una de las grandes dificultades en la sostenibilidad del sistema es la tendencia a formular sin agotar instancias iniciales de tratamiento. Es importante tener un muy buen nivel de evidencia; trabajar en Guías de Atención, consensos y mecanismos colegiados de decisión (entre otras, las juntas médicas, también reglamentadas en la norma); deben considerarse el control por pares y la medición sistemática de los resultados en salud.

El segundo reto es la adaptación: es importante establecer los mecanismos de soporte para que el acto médico no se convierta en una tortura y tenga un efecto de sobrecarga. Siendo humanamente válida la sensación de "no tener tiempo para ver al paciente, pues en veinte minutos no alcanzo a hacer historia clínica, anamnesis, registros, fórmulas y fuera de todo aplicativo web”, es también trascendente decir que la responsabilidad médica está en que el paciente mejore de la manera más efectiva y rápida. Hay un adagio jurídico-médico que reza: "es más negativo no entregar al paciente lo que es, por temor a lo que no ha pasado o peor aún por el dolo de no buscarlo". Busquemos alternativas para optimizar el tiempo, pero pensemos en los pacientes. 
El tercer reto es que quede claro el plan B y hasta el plan C, en caso de falla del procedimiento de formulación y registro. La probabilidad de que el aplicativo WEB falle (sobre todo al inicio) es muy alta. Por esto los registros manuales en los formatos dispuestos por el Ministerio deben tenerse a mano y el proceso debe ser claro.

El cuarto reto: los endocrinólogos adscritos a compañías de seguros de salud y prepagadas, y que hacían los formatos CTC a las EPS para que ellas autorizaran el medicamento, insumo o procedimiento, deben pedir aclaración por escrito a las prepagadas y compañías de seguros acerca del procedimiento futuro. En teoría ya no va a ser tan sencillo.

Es importante aclarar que los ranking de prescriptores mayores de lo no cubierto por el plan de beneficios y las posibles consecuencias negativas mediáticas y legales no están dentro de la agenda pública. Obviamente existirán casos que por el desatino clínico, la falta de evidencia o el dolo pueden ser estudiados; pero en esencia los factores protectores están sustentados en la base científica de la decisión, en el registro adecuado en la historia clínica y en gran parte en la toma de decisiones colegiadas.

Por último, es importante aclarar que las amenazas que a veces se han evidenciado en torno a que lo glosado o las dificultades financieras secundarias a la formulación por fuera del plan de beneficios van a ser cobradas solidariamente a los médicos, es un mecanismo que puede rayar con la ilegalidad y viola el derecho a la salud y a la autonomía. Una obligación de gremios y sociedades es proteger a los médicos en esos casos de condiciones leoninas en los contratos comerciales o laborales entre profesionales y prestadores.

En resumen, las normas, reglas y supuestos del sistema de salud van a requerir un proceso de adaptación que a veces va a ser doloroso. Busquemos el autocontrol sin perjudicar al paciente; pidan ayuda y soporte: existen "mesas de ayuda" de empresas muy bien cimentadas que van a dar soporte en todo el proceso de adaptación. Y ante todo, adaptémonos y entreguemos al paciente lo que es en el momento que es; al fin y al cabo es el sistema de salud de nuestros hijos y familiares... jah! y el de nosotros mismos cuando estamos en el papel de pacientes.

\section{Roberto Baquero Nieto}

$\mathrm{Md}, \mathrm{Mba}$, Phd $(\mathrm{C})^{* *}$

Editorialista invitado 